\title{
Ultrafast Laser Patterning of Thin Films on 3-D Shaped Surfaces for Strain Sensor Applications
}

\author{
Jan Friedrich DUESING, Oliver SUTTMANN, Juergen KOCH, Uwe STUTE and Ludger OVERMEYER
}

\author{
Laser Zentrum Hannover e.V., Hollerithallee 8, 30419 Hannover, Germany \\ Email:j.duesing@lzh.de
}

\begin{abstract}
A femtosecond laser patterning process of thin film sensors on component surfaces is reported. This method is particularly useful for patterning of strain sensors which are sputter deposited directly on curved surfaces. The ablation behavior of $\mathrm{NiCr}$ film irradiated by femtosecond laser pulses at non-normal angles of incidence is modeled and experimentally verified with linear and circular beam polarization for incidence angles up to $80^{\circ}$. It is shown that the ablation threshold behavior can be described when including polarization and angle dependent Fresnel reflection into the laser ablation model. The laser process is finally demonstrated by patterning $\mathrm{NiCr}$ thin film sensors on a non-planar surface of a mechanical component for a machine tool.
\end{abstract}

DOI:10.2961/jlmn.2012.03.0014

Keywords: femtosecond laser pulses, thin film processing, ablation threshold, incident angle, polarization, $\mathrm{NiCr}$, surface strain sensor

\section{Introduction}

Thin film strain sensors directly deposited onto the surface of mechanical components enable measurement of forces, pressure or strain in harsh environments and at high temperatures [1-6]. High-resolution thin film sensors are typically structured by standard lithographic processes on a wafer level. However, lithography is limited to planar surfaces and often cost-effective only for large batch sizes. We have developed an alternative route for patterning of strain sensors on component surfaces by using a direct laser ablation process $[7,8]$. A focused laser beam is scanned across the surface and selectively removes the sensing layer with a spatial resolution of $\sim 20 \mu \mathrm{m}$ in order to form the sensor geometry. Thermal induced damage of the underlying layers is suppressed by using ultrashort laser pulses.

The direct writing approach using a laser beam as a patterning tool on a component surface has some advantages compared to mask-based techniques. For example, photolithographic patterning requires the manufacturing of a photo mask as well as a series of processing steps to finalize a sensor pattern, e.g. deposition of photo resist, light exposure, etching, and resist stripping. The direct write approach reduces the number of process steps and the sensor geometry is written directly from a computer model. Furthermore, it enables patterning of film sensors on $3 \mathrm{D}$ shaped surfaces, which clears the way for many novel applications of thin film strain sensors, e.g. on drive shafts or complex machine components.

The machining quality of ultrafast laser ablation processes can be optimized at laser intensities slightly above the ablation threshold since thermal damaging of remaining material is minimized $[9,10]$. At the same time, high repetition rate lasers in combination with fast laser scanners are used to achieve high processing speeds. During laser scanning of curved surfaces the angle of incidence varies with the topography of the component. Hence, reflectivity and energy density on the surface change with angle of incidence and the polarization of the laser beam. In this work, we investigate the effect of non-normal angle of incidence as well as beam polarization on ablation thresholds during laser thin film processing.

\section{Laser Thin Film Ablation on Inclined Surfaces}

Interaction of metallic thin films with ultrashort laser pulses is generally characterized by threshold behavior. The threshold fluence is the value of energy per area which separates two distinguishable laser-induced effects, e.g. surface modification and ablation. In this work, we define the ablation threshold as the fluence which is necessary to completely remove the film from the substrate (measured with optical methods).

Due to reflection losses on the film surface only a fraction of the irradiated fluence is absorbed within the film and initiates film removal. The absorbed fluence is in most cases not directly accessible by measuring methods, and thus, can only be estimated by comparison to an ablation model including reflection losses. In the following, we model the ablation behavior by considering angle and polarization dependent Fresnel reflection. Further parameters which may influence the ablation threshold, such as pulse duration, number of pulses (incubation effect), surface roughness, or film thickness, are not the scope of this work.

Starting with the situation of a focussed laser beam with a single-mode Gaussian-shaped beam profile at normal incidence; the local fluence on a surface can be described as:

$$
F(r)=F_{0} \cdot \exp \left(-2 \frac{r^{2}}{w_{0}{ }^{2}}\right)
$$

$F_{0}$ is the peak fluence in the center of the beam and $w_{0}$ is the beam waist at $1 / \mathrm{e}^{2}$ of the peak fluence. The pulse 


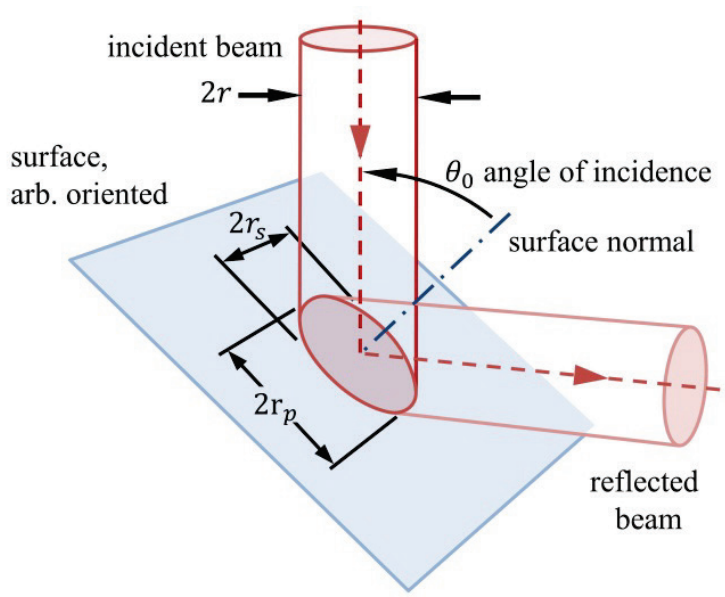

Fig. 1: Projection of a circular laser beam (small divergence) on an inclined surface results in a nearly elliptical irradiation area.

energy can be calculated by integrating the fluence over the entire area. If pulse energy $E_{p}$ and the beam waist $w_{0}$ are known the peak fluence can be calculated as:

$$
F_{0}=\frac{2 E_{p}}{\pi w_{0}^{2}} .
$$

In the case of an inclined surface the fluence distribution is described by the cross section of the surface plane with the caustic of the focused laser beam. For laser beams with small beam divergence the irradiated area is nearly elliptical (cf. Fig. 1). The local fluence on the surface can then be approximated as:

$$
F\left(r_{s}, r_{p}\right)=F_{0} \cdot \exp \left(-2 \frac{r_{s}{ }^{2}+r_{p}{ }^{2} \cdot \cos ^{2} \theta_{0}}{w_{0}{ }^{2}}\right) .
$$

The angle of incidence, $\theta_{0}$, is defined as the angle between beam axis and surface normal. Accordingly, the peak fluence is calculated as:

$$
F_{0}=\frac{2 E_{p} \cos \theta_{0}}{\pi w_{0}^{2}} .
$$

The absorbed fluence is calculated from irradiated fluence by including a term for reflection losses, assuming that transmission and scattering effects are negligible:

$$
F_{a b s}=F_{i r r} \cdot(1-R) \text {. }
$$

The reflectivity is calculated from Fresnel's equations:

$$
\begin{aligned}
& R_{s}=\frac{\sin ^{2}\left(\theta_{0}-\theta_{1}\right)}{\sin ^{2}\left(\theta_{0}+\theta_{1}\right)}, \\
& R_{p}=\frac{\tan ^{2}\left(\theta_{0}-\theta_{1}\right)}{\tan ^{2}\left(\theta_{0}+\theta_{1}\right)}, \\
& R_{c}=\left(R_{s}+R_{p}\right) / 2,
\end{aligned}
$$

where $R_{s}$ and $R_{p}$ being the reflectivity for linear polarized light perpendicular and parallel to the plane of incidence, respectively, and $R_{c}$ being the reflectivity for circular polarization. The refracted angle $\theta_{l}$ can be calculated

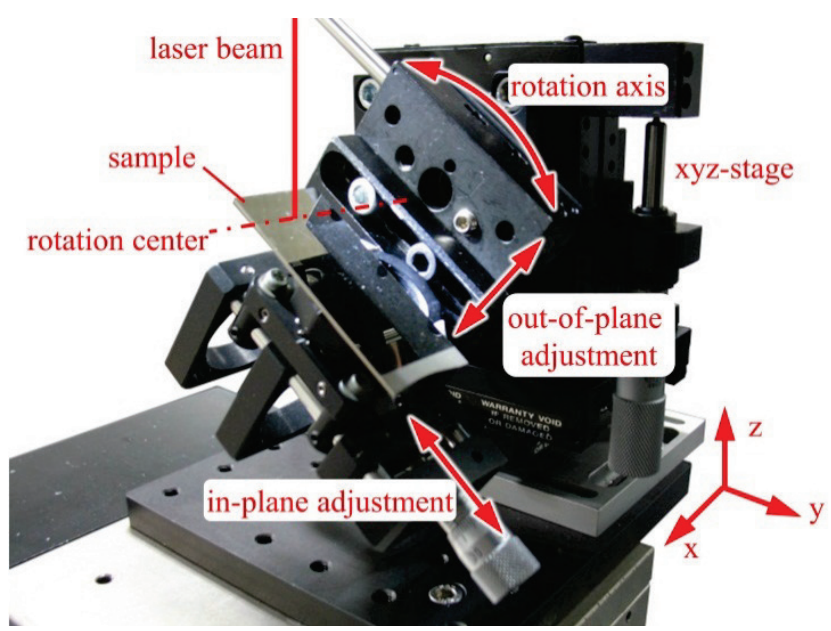

Fig. 2: A 6-axes manual stage is used to align sample and rotation axis with respect to the laser beam in order to bring sample surface, beam focus and rotation center in coincidence.

from Snell's law using $n_{0} \cdot \sin \theta_{0}=n_{1} \cdot \sin \theta_{1}$ with the $(\mathrm{com}-$ plex) refractive indices $n_{0}$ of air and $n_{1}$ of the film material.

\section{Experiment}

\subsection{Substrate Preparation and Handling}

$\mathrm{NiCr}(80 / 20 \mathrm{wt} \%)$ films with a thickness of $200 \mathrm{~nm}$ are deposited by magnetron sputtering directly onto a glass slide. The refractive indices $n_{\text {air }}=1$ and $n_{N i C r}=1.6+2.4 i$ are determined prior to the experiment using reflectivity measurements with a cw laser at $515 \mathrm{~nm}$. The sample is then attached to a multi-axis stage system as shown in Fig. 2. A horizontal rotation axis is used to tilt the sample in order to vary the angle of incidence of the laser beam with respect to the sample surface. In order to prevent focus shift errors the rotation center and the sample surface are both moved into the laser beam focus by translation stages. During the alignment procedure the laser spot on the sample as well as any sample movement during rotation is observed by offaxis camera imaging systems. By proper alignment it is possible to rotate a single spot on the sample around the laser focus point up to $80^{\circ}$ incidence angle.

\subsection{Laser Parameter}

Femtosecond laser pulses with a pulse duration of 0.6 ps (FWHM, measured by autocorrelation method) and a center wavelength of $515 \mathrm{~nm}$ are generated by a diode pumped amplified femtosecond system from Amplitude Systemes (type 's-Pulse HP'). The pulse energy is controlled by a motorized half-wave plate and polarizing beam splitter. The polarization state of the laser beam is modulated by a half-wave plate and a quarter-wave plate. The laser beam is focused by a lens with $100 \mathrm{~mm}$ focal length. A variable beam expander in front of the lens is used to adjust the laser focus spot to $25 \mu \mathrm{m}$ (diameter at $1 / \mathrm{e}^{2}$ of peak fluence). The beam profile is Gaussian shaped in $\mathrm{TEM}_{00}$ mode. Ablation experiments are carried out by pulsing 10 laser pulses on the same sample spot. All experimental parameters are summarized in Tab. 1. 


\subsection{Image Analysis of Ablation Area}

The ablation craters are imaged by transmission microscopy using a 100x objective lens. In the transmission images the ablated area appears light while intact film is dark. Care is taken to choose a proper exposure time in order to prevent the camera chip from saturation in the lighter regions which would cause edge blurring effects, and on the other side, still gives enough contrast for the subsequent image processing. An image analysis algorithm is developed which allows automated measurement of major and minor axes of elliptically shaped ablation areas. It enables fast analysis of a large amount of images (more than 720 images of ablation craters are collected for this experiment) as well as robust measurement of ablated areas even with indistinct crater edges.

The image analysis procedure is as follows: In a first step, the image intensity counts are summarized along one axis of the ablated ellipse which is aligned parallel the image border. Assuming that the ablated area has an elliptical shape with a center point in the image at position $\left(x_{0}, y_{0}\right)$ and with axis $\left(r_{x}, r_{y}\right)$, then a peak is formed in the curve at position $x_{0}$ and with a peak width of $2 \cdot r_{x}$. In the next step the resulting curve is fitted to the following elliptic equation:

$$
I(x)= \begin{cases}\alpha \cdot \sqrt{1-\left(\frac{x-x_{0}}{r_{x}}\right)^{2}}+\beta, & \left|\frac{x-x_{0}}{r_{x}}\right|<1 \\ \beta, & \left|\frac{x-x_{0}}{r_{x}}\right| \geq 1\end{cases}
$$

$r_{x}$ and $x_{0}$ as well as the scaling factor $\alpha=2 \cdot r_{y} \cdot I_{I}$ and offset $\beta=h \cdot I_{0}$ are used as variable fitting parameters. $I_{0}$ and $I_{1}$ are the mean intensity counts of background and transmitted light, respectively, and $h$ is the image height in pixels. In order to extract the other axis the procedure is repeated with the image rotated by $90^{\circ}$.

\section{Results and Discussion}

An exemplary selection of transmission images of ablated thin film samples is shown in Fig. 3. The ellipticity of the ablated area increases with an increasing $\theta_{0}$. Image integration curves are shown for a sample at $\theta_{0}=70^{\circ}$ as solid lines. From these curves minor and major are extracted by fitting to Eq. (7). This procedure is repeated for all collected transmission images and the resulting (squared) ablation radii for major and minor axes are plotted versus the pulse energy. Fig. 4 shows for better clarity only a selection of measured data at circular polarization.

By rearranging Eq. (3) and (4) the relationship between squared radii for minor and major axis from pulse energy is obtained, following to route of Liu [11]:

$$
\begin{aligned}
& r_{\text {minor }}^{2}=\frac{1}{2} w_{0}^{2} \ln \left(\frac{E_{p}}{E_{t h}}\right), \\
& r_{\text {major }}^{2}=\frac{w_{0}^{2}}{2 \cos ^{2} \theta_{0}} \ln \left(\frac{E_{p}}{E_{t h}}\right) \text {. }
\end{aligned}
$$

Tab. 1: Overview of laser parameters.

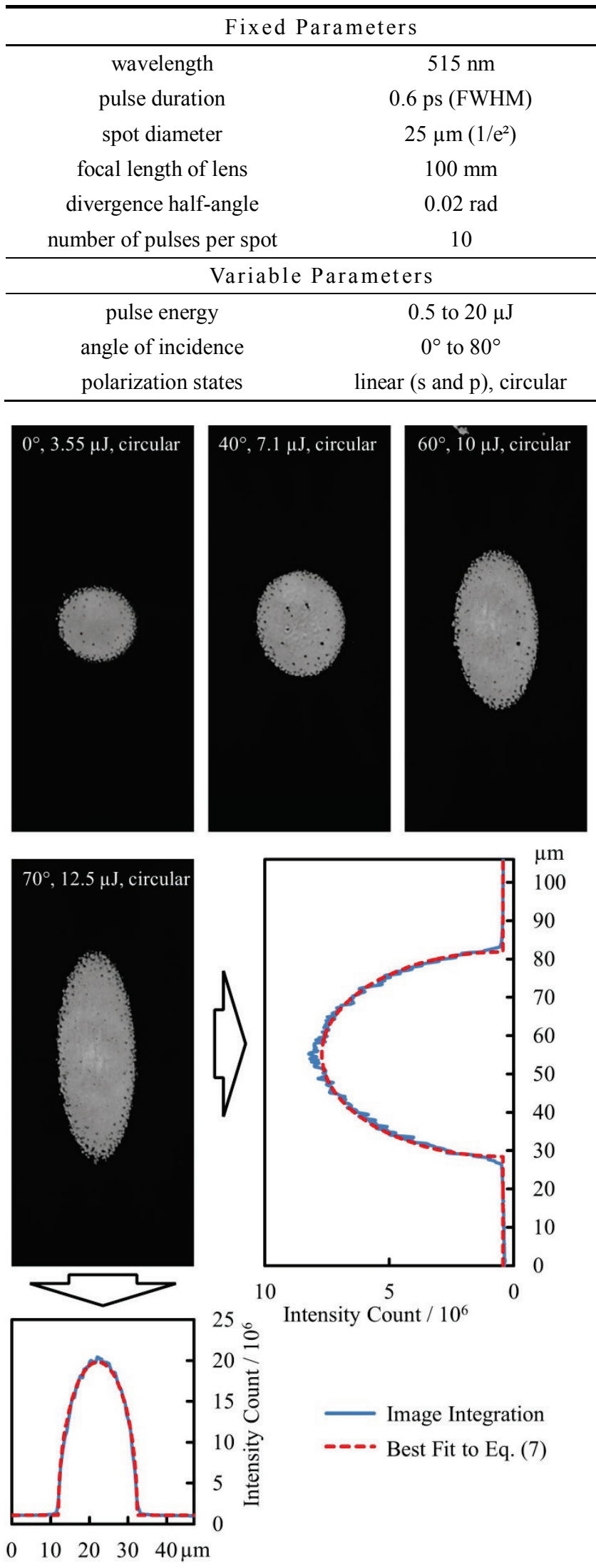

Fig. 3: Transmission microscopy images of ablated $\mathrm{NiCr}$ film on glass substrate. With increasing incidence angles the ablated area becomes more and more elliptical. The radii of the ellipse is determined by summarizing the intensity counts along one image axis and fitting the resulting curve to Eq. 7 . 


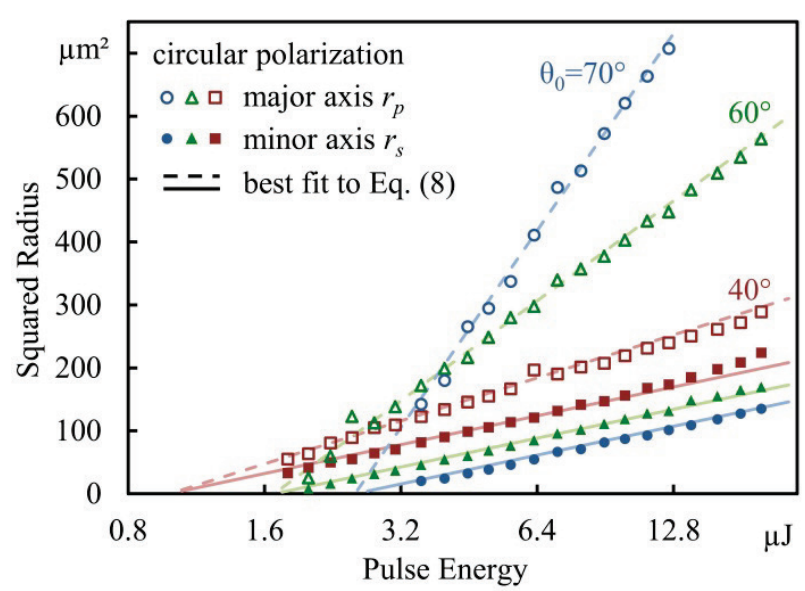

Fig. 4: For Gaussian beam profiles the squared radii of ablated area depends logarithmic from laser pulse energy. The ablation threshold is determined by interpolating the best-fit lines to the intersection point with the $\mathrm{x}$-axis.

The best fit to these functions is shown as lines in Fig. 4. Two parameters are extracted from these fits: (i) the beam waist parameter $w_{0}$ is calculated from the slope of the lines, and (ii) the threshold pulse energy $E_{t h}$ is extracted from the interception point of the best-fit lines with the $\mathrm{x}$ axis. The pulse energy is then converted to irradiation fluence using Eq. (4) based on a best-fit beam diameter $2 w_{0}=23 \mu \mathrm{m}$.

All determined threshold fluences for three polarization states and incidence angles between $0^{\circ}$ up to $80^{\circ}$ are summarized in Fig. 5. The error bars represent the estimated error which is extracted from the covariance matrix during the fit procedure. It can be seen that the ablation threshold behavior for all polarization states is similar at low $\theta_{0}$ but differ significantly at high $\theta_{0}$. The solid lines represent a best-fit result to Eq. (5) yield an absorbed fluence $F_{a b s}=$ $0.19 \mathrm{~J} / \mathrm{cm}^{2}$. The good agreement of Eq. (5) to measured data within the error bars lead to following statements:

1. The threshold fluence for ablation of thin $\mathrm{NiCr}$ film under non-normal $\theta_{0}$ up to $80^{\circ}$ can be qualitatively described by a threshold model which includes a term for reflection losses on the surface of the film (Eq. 5).

2. Modeling ultrafast laser ablation based on optical parameters for $\mathrm{cw}$ radiation may result in inaccurate ablation threshold estimation. Especially in the medium intensity range of ultrashort laser pulses near the ablation threshold $\left(10^{11}\right.$ to $\left.10^{14} \mathrm{~W} / \mathrm{cm}^{2}\right)$ the reflectivity of metals may not be comparable to $\mathrm{cw}$ reflectivity anymore. E.g. for copper it has been shown that the reflectivity drops at intensities of $\sim 10^{13} \mathrm{~W} / \mathrm{cm}^{2}$ during the interaction of a $150 \mathrm{fs}$ laser pulse at $800 \mathrm{~nm}$ [12]. This effect has been attributed to the large heating rates of absorbing electrons and accompanied highly non-equilibrium states of the irradiated metal. On the other hand, the presented method is based on measuring ablation area created with various pulse energies but at constant fluence, namely the ablation fluence. Thus, any fluence dependency of absorption coefficient, e.g. in the center of the irradiated area, is not present in the data. Assuming an

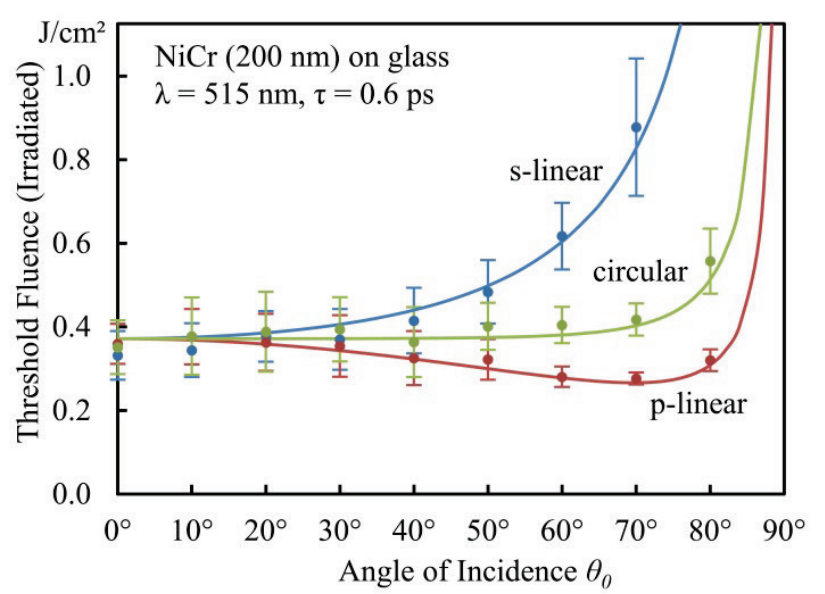

Fig. 5: Ablation threshold versus angle of incidence for three polarization states. The solid lines represent a best fit to Eq. (5).

increased absorption for $\mathrm{NiCr}$ during the laser pulse interaction, then the quantitative value for $F_{a b s}$ might be slightly underestimated but the qualitative shape of the curve in Fig. 5 will not be significantly affected.

3. In terms of a practical application: For processing of $\mathrm{NiCr}$ films circular polarization would be preferred at $515 \mathrm{~nm}$ wavelength since the ablation threshold is constant over a wide range of $\theta_{0}$ up to $70^{\circ}$ and furthermore independent from surface orientation with respect to a polarization axis. This simplifies the thin film patterning process as no additional correction for reflectivity dependency at varying incident angles is required.

\section{Application: Sensor Patterning on Curved Surfaces}

As demonstrated, ultrashort laser pulses can be used to ablate thin metal films from highly tilted surfaces. This is of high practical relevance for manufacturing of thin film sensors on component surfaces. It enables integration of sensors at positions which cannot be processed with standard lithographic patterning methods based on planar masks.

Fig. 6 shows NiCr thin film strain sensors which are laser patterned into a v-shaped groove of an aluminum component. Prior to the laser patterning process the component is sputtered with an insulating $\mathrm{Al}_{2} \mathrm{O}_{3}$ layer $(3 \mu \mathrm{m})$ and a sensing NiCr film $(200 \mathrm{~nm})$. The NiCr layer is then selectively ablated with a circular polarized laser beam on a micromachining setup using a xyz galvanometer scanning system and a telecentric f-theta focusing optic. Several lines around the contour of the geometry are scanned (dark area in Fig. 6), creating electrically isolated sensor patterns. Each pattern has a meandering and strain sensitive resistance path as well as two pads for electronic contacting. The focus position is kept fix at the bottom of the radius and no variable pulse energy controller is used. Though, the patterning process is based on serial ablation strategy the overall processing time for each resistor is less than 15 seconds at a pulse repetition rate of $10 \mathrm{kHz}$.

The change of electrical resistance upon mechanical load has been tested elsewhere [8] on tensile specimen and it has been shown that the thin film sensors have a linear response to an applied force. 

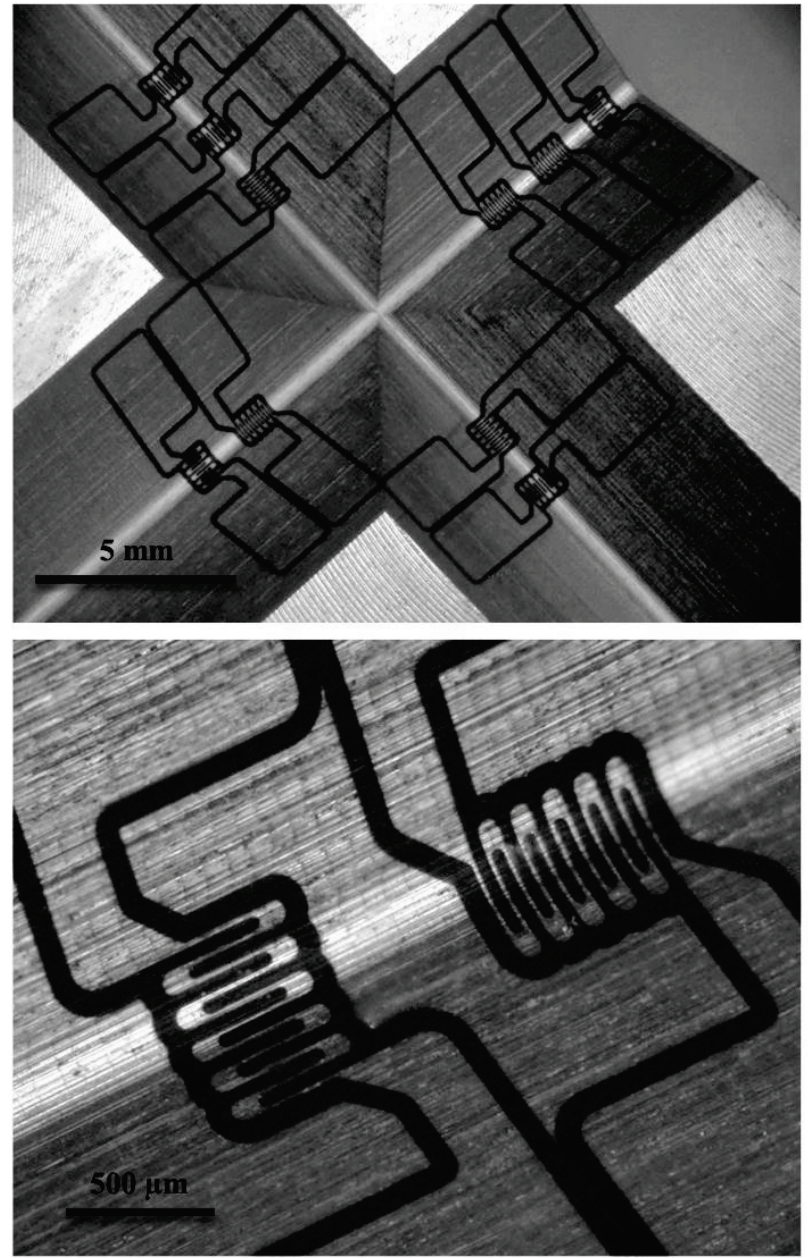

Fig. 6: Laser patterned thin film strain sensors processed into a vshaped groove of an aluminum component. The top metallic layer is selectively removed by ultrashort laser pulses (dark area).

\section{Summary}

Ultrafast laser ablation of thin NiCr films on component surfaces under non-normal angle of incidence is demonstrated. It is shown that the ablation process depends on the angle of incidence and on the polarization state of the laser beam. A model for laser pulse ablation has been developed which includes reflection dependencies at inclined surfaces. The model is verified in ablation experiments of $\mathrm{NiCr}$ films on glass substrates for a broad range of incidence angles (normal incidence up to $80^{\circ}$ ) and for three polarization states.

The laser ablation process has practical significance for patterning of thin film electronics or sensors on component surfaces. It is an alternative route to standard photolithographic patterning processes. Due to its direct-write approach it enables patterning of sensors on arbitrary curved surfaces and may enable novel concepts for intelligent sensor integration on component surfaces, e.g. in machine tool components for online process monitoring.

\section{Acknowledgments}

The German Research Foundation (DFG) is gratefully acknowledged for financial support within the Collaborative Research Centre CRC 653, "Gentelligent Components in their Lifecycle".

\section{References}

[1] P. Kayser, J.C. Godefroy and L. Leca: Sensor. Actuat. A-Phys., 37-38, (1993) p.328-332.

[2] S, Biehl, S. Staufenbiel, F. Hauschild and A. Albert: Microsyst. Technol., 16:5, (2011) p.879-883.

[3] L.C. Martin, J.D. Wrbanek and G.C. Fralick: Proc. 19th Int. Congr. on Instrumentation in Aerospace Simulation Facilities ICIASF, Cleveland, (2001) p.196203.

[4] J.-F. Lei and H.A. Will: Sensor. Actuat. A-Phys., 65 (1998) p.187-193.

[5] Y. Mihara and T. Someya: Sensors, Proc. of IEEE (2002) p.954-959.

[6] J. Longtin, S. Sampath, S. Tankiewicz and R.J. Gambino: IEEE Sens. J., 4:1, (2004) p.118-121.

[7] O. Suttmann, J.F. Duesing, U. Klug and R. Kling: J. Laser Micro. Nanoen., 6:1, (2011) p.31-36.

[8] L. Overmeyer, J.F. Duesing, O. Suttmann and U. Stute: CIRP Ann., 61:1, (2012) p. 215-218.

[9] J. Meijer, K. Du, A. Gillner, D. Hoffmann, V.S. Kovalenko, T. Masuzawa, A. Ostendorf, R. Poprawe and W. Schulz: CIRP Ann., 51:2, (2002) p.531-550.

[10]F. Siegel, U. Klug, R. Kling: J. Laser Micro. Nanoen., 4:2, (2009) p.104-110.

[11] J.M. Liu: Opt. Lett., 7:5, (1982) p. 196-198

[12]S.E. Kirkwood, Y.Y Tsui, R. Fedosejevs, A.V. Brantov and V.Y. Bychenkov: Phys. Rev. B, 79, (2009) 144120.

(Received: June 29, 2012, Accepted: September 27, 2012) 